\title{
Performance Analysis of Distributed Reservation Protocol for UWB-Based WPAN
}

\author{
Kuang-Hao Liu, Member, IEEE, Xuemin (Sherman) Shen, Senior Member, IEEE, \\ Ruonan Zhang, and Lin Cai, Member, IEEE
}

\begin{abstract}
In this paper, we propose an analytical model for the distributed reservation protocol (DRP), which is defined in the WiMedia specification for ultra-wideband (UWB)-based wireless personal area networks (WPANs). We model the tagged user as a discrete-time queue with vacations, which captures the joint behavior of a queue length variation and a time-varying UWB channel due to shadowing under a given reservation pattern. Furthermore, we consider two reservation methods: hard reservation and soft reservation. With the hard reservation, a time slot is exclusively used by the owner, whereas the unused time slots can be accessed by other users using the soft reservation. Closed-form expressions of important performance metrics such as the mean service time, the waiting time, and the throughput are derived. Through numerical results, we validate the accuracy of the proposed analytical model and investigate the interaction between the DRP and various system parameters. This paper should provide insights into the performance of the DRP and useful guidelines to further improve the protocol to support isochronous applications with a tight delay requirement in UWB-based WPANs.
\end{abstract}

Index Terms-Channel model, distributed reservation protocol (DRP), matrix-geometric approach, shadowing, ultra-wideband (UWB), vacation queue, wireless personal area network (WPAN).

\section{INTRODUCTION}

$\mathbf{U}$ LTRA-WIDEBAND (UWB) is an appealing technology for short-range bandwidth-demanding wireless applications [1], [2]. With a data rate of several hundreds of megabits per second, UWB networks can simultaneously support multiple isochronous streams such as high-definition television, voice over Internet protocol, and interactive gaming [3]. Research efforts from both academia and industry have been devoted to enabling various emerging multimedia applications

Manuscript received December 10, 2007; revised April 6, 2008 and June 16, 2008. First published July 16, 2008; current version published February 17, 2009. This paper was presented in part at the IEEE International Conference on Communications, Beijing, China, May 2008. This work was supported in part by the Natural Sciences and Engineering Research Council of Canada under Grant STPSC 356682. The review of this paper was coordinated by Prof. R. Jantti.

K.-H. Liu and X. Shen are with the Centre for Wireless Communications, Department of Electrical and Computer Engineering, University of Waterloo, Waterloo, ON N2L 3G1, Canada (e-mail: k8liu@ @bcr.uwaterloo.ca; xshen@bbcr.uwaterloo.ca).

R. Zhang and L. Cai are with the Department of Electrical and Computer Engineering, University of Victoria, Victoria, BC V8W 3P6, Canada (e-mail: rzhang@ece.uvic.ca; cai@ece.uvic.ca).

Color versions of one or more of the figures in this paper are available online at http://ieeexplore.ieee.org.

Digital Object Identifier 10.1109/TVT.2008.928638 in the wireless personal area network (WPAN) based on UWB technologies [4], [5].

The WiMedia Alliance has recently launched its physicallayer and medium-access-control (MAC) protocol specifications using the multiband orthogonal frequency-division multiplexing (MB-OFDM) UWB technology [6]. To achieve ad hoc connectivity, the WiMedia specification defines two distributed channel access mechanisms - the prioritized channel access (PCA) and the distributed reservation protocol (DRP). The PCA is a contention-based protocol, which provides differentiated channel access via the mechanisms that are similar to the enhanced distributed channel access (EDCA) in IEEE 802.11e. Devices first sense the channel before accessing the medium, and class-dependent contention parameters are used to prioritize traffic classes. It is known that the quality-ofservice (QoS) guarantee in the EDCA is achieved in a statistical manner, which makes it difficult to satisfy the stringent delay requirement of isochronous traffic [7]. The detailed analysis of the PCA can be found in [8] and [9].

The DRP is a distributed time-division multiple-access (TDMA) protocol, by which users have the exclusive right of transmission during the reserved time slots. Different from the ordinary TDMA, negotiations of channel time are carried out between peer users through a distributed negotiation procedure. Such a reservation-based protocol provides guaranteed transmission time and, thus, is preferable to isochronous traffic with stringent delay requirement. However, isochronous traffic reveals bursty characteristics, where the packet interarrival time is nonuniform. Consequently, the discrepancy between the reserved bandwidth and the time-varying bandwidth requirement may lead to the inefficient utilization of network resources when the reservation is made in a hard sense. This disadvantage of the DRP can be alleviated through the soft reservation by which other backlogged users can access the unused slots according to the access rule of the PCA.

While the centralized TDMA protocol and its variants have been studied extensively [10]-[12], the distributed slot reservation protocol has been explored less. The main difference of the DRP from its centralized counterpart lies in the resultant reservation pattern. To reduce the delay variation, it is desirable to reserve contiguous or uniformly spaced time slots per scheduling cycle (i.e., superframe), which is possible with the aid of a centralized coordinator. However, maintaining such a uniform reservation pattern (a uniform interval between two reservations) is difficult in a distributed environment, where the available time slots within a scheduling cycle may reveal an arbitrary pattern. In [13], the delay performance of the 
DRP under a given reservation pattern is analyzed using a 2-D embedded Markov chain based on the assumption of Poisson arrivals. Although the model is straightforward, it is limited to deal with the hard reservation only and not applicable to real-time traffic with bursty arrivals. On the other hand, the assumption of an error-free wireless channel ignores the impact of time-varying wireless channel impairments on the delay performance, which have been noticed in many recent studies for typical wireless networks [14]-[17].

In an indoor environment, UWB devices are likely to be stationary, while nearby people may frequently walk through the line of sight (LOS) of an ongoing link, resulting in the peopleshadowing effect. For UWB communications with a data rate of several hundreds of megabits per second, half-second shadowing can affect more than hundreds of megabits of data and, thus, severely degrade the quality of the ongoing isochronous streams, even with granted channel time for transmissions.

In this paper, we propose an analytical model for the DRP considering a specific scenario, where the communication link is affected by people shadowing, and the reserved time slots are nonuniformly distributed in the superframe. Based on the measurement results, the UWB multipath channel with shadowing is modeled as a finite-state discrete-time Markov chain at the packet level. In addition to the time-varying channel behavior, the use of a reservation method and the induced reservation pattern also affect the protocol performance. Given a reservation pattern, we are interested in the impact of the reservation method on the DRP performance. The proposed analytical model is developed based on the vacation queuing model, where the interval between two reservation periods is regarded as the vacation period of the tagged user.

The main contributions of this paper are threefold. First, we propose an analytical model that can capture the dynamic behaviors of different layers across the protocol stacks. To the best of our knowledge, this is the first paper analyzing the DRP protocol considering the characteristics of time-varying UWB channels. Second, by using the matrix-geometric approach, we solve the complex queuing system and obtain several important performance metrics in closed-form expressions, such as the mean service time, the waiting time, and the throughput. Third, through numerical results, we can obtain insights into the DRP performance and provide useful guidelines to further improve the protocol.

The remainder of this paper is organized as follows. Section II presents the protocol description and the modeling approach for the DRP, followed by the packet-level UWB shadowing channel model. We formulate the queuing system and derive various performance metrics for hard and soft reservations in Section III. Numerical results and discussions are given in Section IV. This paper is concluded in Section V.

\section{PRELIMINARIES}

The system under investigation is a distributed UWB WPAN, where users reserve time slots using the DRP. Because of the movement of nearby people, an ongoing link may be frequently shadowed off. Our goal is to quantitatively assess the protocol performance under the people shadowing effect, considering soft and hard reservation methods. In this section, we first give an overview of the DRP and explain how the protocol behavior can be modeled given a specific reservation pattern. We then present the methodology of modeling the UWB shadowing channel at the packet level.

\section{A. Overview of the DRP and Its Modeling}

In WiMedia MAC, the channel time is divided into superframes composed of 256 media-access slots (MASs). A superframe consists of a beacon period (BP) and a data transfer period (DTP), as depicted in Fig. 1. In the BP, each user transmits its own beacon frame, containing timing and control information to allow fully distributed channel access. The BP is followed by a DTP, in which users communicate with each other through either the contention-based PCA or the reservation-based DRP, or the combination of both. In the DTP, a number of MASs should be retained for PCA traffic. The remaining MASs are available for reservation via the DRP. To reserve MASs, a user needs to first send a request, indicating the set of MASs to be reserved, to its intended receiver (Rx). The request can be encoded in the beacon frame or sent via the PCA or the DRP. Upon receiving the request, the Rx checks the availability of the intended MASs according to its locally collected information. The reservation can be successfully made only when resources are available at the sender and the $\mathrm{Rx}$ sides, that is, devices within the transmission range cannot reserve the same time slot. If the reservation is accepted, the $\mathrm{Rx}$ announces the result in its beacon. Consequently, other users become aware of the reservation, and the sender can start the transmission from the next superframe using the reserved MASs. Moreover, time slots can be reserved in a hard or soft manner. By a hard reservation, the channel is occupied by the owner throughout the reserved MASs. If a soft reservation is used, the unused MASs can be accessed by other users following the PCA rule. The reservation type is indicated in the result of reservation.

Fig. 1 shows an exemplary superframe that is seen from a tagged user. A tagged user is said to be in service during its reserved time slots; otherwise, it is said to be on vacation. Therefore, the vacation time of a tagged user is equivalent to the union of the reserved time slots of other competing users, the unreserved slots, the beacon time, and the period that is retained for PCA traffic in a superframe. Since the unreserved slots can be derived given the reservation pattern, we focus on the service period of a tagged user. The length of a service period depends on the reservation method. When the hard reservation is used, the service period is identical to the reservation period, which is deterministic. On the other hand, the service period in the soft reservation may be less than or equal to the reservation period, depending on the buffer status.

Because of the difference between the reservation methods, modeling the vacation period may not be trivial. In principle, the vacation period can be described by the discrete-phase (PH) type distributions. To elaborate on this, we first briefly review the discrete-PH-type distribution. Generally, a discretePH distribution characterizes the time until absorption into state $K$ in a Markov chain on the state space $\{0, \ldots, K\}$ with initial probability vector $\left(\boldsymbol{\eta}, \eta_{K}\right)$, where $\eta_{K}=1-\boldsymbol{\eta} \mathbf{1}$, with $\mathbf{1}$ being 


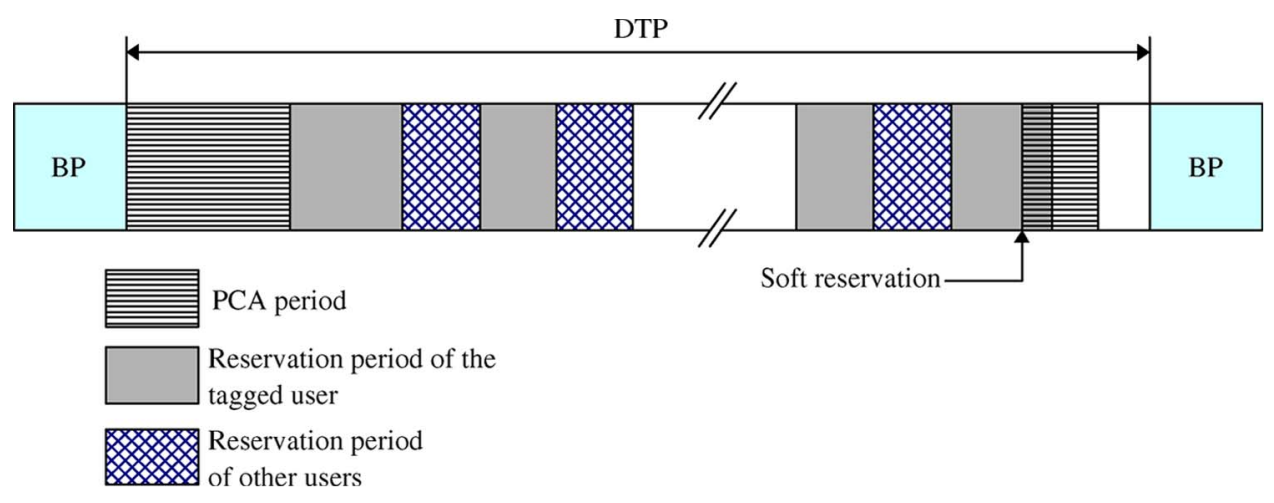

Fig. 1. Superframe structure defined in the WiMedia MAC protocol.

a column vector of all ones, and the transition matrix is structured by

$$
\left[\begin{array}{cc}
\mathbf{T} & \mathbf{T}^{0} \\
\mathbf{0} & 1
\end{array}\right]
$$

where $\mathbf{T}^{0}=\mathbf{1}-\mathbf{T} 1$. Thus, the knowledge of $\eta$ and $\mathbf{T}$ is sufficient to characterize a discrete-PH distribution, which is denoted by $\mathrm{PH}_{d}(\boldsymbol{\eta}, \mathbf{T})$ with order $K$. The discrete-PH-type distribution is general enough to represent every discrete distribution with finite support. For example, a geometric distribution with parameter $p$ can be represented by $\mathrm{PH}_{d}(1,1-p)$ with order 1.

If the service period of an individual user obeys a discrete$\mathrm{PH}$ distribution, then the vacation period of the tagged user, which is the sum of the service periods of other users, can also be represented by the convolution of multiple discrete-PH distributions. For the case of the hard reservation, the service period of each user is deterministic, which is a special case of the discrete-PH distribution. For the soft reservation, the service period is stochastic, depending on the queue status. Once the queue is empty, the channel becomes idle, and thus, other users can immediately access the channel following the PCA principle. In this case, the tagged user queue can be modeled by a vacation queue with a time-limited policy. Such a vacation queue has been studied in [18], where the service period under the time-limited vacation policy is shown to be of the discrete$\mathrm{PH}$ type. Notice that if we further consider the other period in which the tagged user is not transmitting data, such as the BP and the time allocated to the PCA in each superframe, the resultant vacation time remains PH-type distributed, considering the facts that the deterministic variable is a special case of a discrete-PH-type distribution, as well as the closure property of a PH-type distribution. In an attempt to study the interaction between the time-varying channel and the queue variation, we focus on modeling the joint behavior of these two dynamics and assume that the vacation-time distribution of a given reservation pattern is known in the sequel. The derivation of the vacationperiod distribution is presented in Appendix I.

\section{B. Packet-Level Channel Model for UWB Shadowing}

In indoor environments, the time variation of wireless channels may be caused by two types of multipath fading-user mobility and shadowing. For typical UWB indoor applications, the transmitter (Tx) and the Rx are more likely to be stationary. Thus, we consider the case when the time variation in the received signal power is mainly due to people shadowing.

To evaluate the actual protocol performance for UWB applications, a channel model that captures the multipath and time-varying characteristics of UWB propagation is a crucial component in the entire system modeling. The IEEE standardization group has suggested four types of UWB indoor channel models (CM-1 to CM-4) for system testing and validation [19]. However, such a waveform simulation needs to simulate the transmission of every symbol over the wireless channel, which is time-consuming for the sake of upper layer protocol analysis. Rather, a packet-level channel model that provides the packet error rate (PER) information according to statistical channel properties can greatly facilitate system modeling. In [20], a packet-level model for a UWB channel considering the people shadowing effect is proposed. We briefly introduce how to obtain the packet-level channel model in the following.

The effect of shadowing can be estimated based on the angular power spectrum (APS) [21]. When a person (i.e., a scatter) steps toward the LOS of an ongoing link, certain angular ranges are shadowed off such that only partial transmission power can be received at the Rx end. The APS describes the distribution of power over the incident angle. As the multipath components may arrive with different delays, the APS of the UWB signal denoted by $\mathbb{P}(\theta, \tau)$ is a function of the tap delay, which is given by

$$
\mathbb{P}(\theta, \tau)= \begin{cases}\operatorname{rect}\left(\frac{2 \pi \tau}{\tau_{\max }}\right), & 0<\tau<\tau_{\max } \\ \frac{1}{2 \pi}, & \tau \geq \tau_{\max }\end{cases}
$$

where $\theta$ is the incident angle with respect to the LOS component, and $\tau$ is the delay of the tap. Based on the APS of the UWB propagation, we can obtain the shadowed channel impulse response (IR) based on a simple scenario that is employed in [22] for measuring the people shadowing effect. A person, which is emulated as a cylinder with a radius $r$, moves through the LOS between the Tx and the Rx at an average speed of $v$. The moving path is assumed to be perpendicular to the LOS, with the initial point $d$ distance away from the LOS. Based on this scenario, Zhang and Cai [20] have proposed the procedure of obtaining the shadowed channel IR. 
We then can construct a channel model to describe the dynamic behavior of the UWB shadowing channel at the packet level. Similar to the notion of the finite-state Markov chain channel model that is widely used in modeling the Rayleigh fading channel, a Markov chain is used to characterize the channel variation by two statistics - the state transition probability and the state-dependent PER. To obtain the state transition probability, we first divide the area around the LOS into $N$ zones. The spatial partition is symmetric with respect to the LOS, resulting in a Markov chain with state space $\{x, x=0$, $\ldots, 2 N\}$, where each state corresponds to a spatial zone, and state 0 represents the spatial zone without shadowing. The average duration that the channel state remains at state $n$, i.e., $t_{n}$, is decided by the area of the partition zone and average mobility speed $v$. In the corresponding discrete-time Markov chain, each channel state will last for $N_{n}=t_{n} / t_{p}$ packet durations, where $t_{p}$ is the packet transmission time. Let the time of a user staying within each zone be geometrically distributed with mean $N_{n}$. The transition probability of the discrete-time Markov chain can, thus, be approximated as

$h_{x, y}= \begin{cases}\lambda_{0}, & x=0, y=1 \\ 1-\lambda_{0}, & x=y=0 \\ \frac{1}{N_{x}}, & x \in(1, \ldots, 2 N), y=\bmod (x+1,2 N+1) \\ \frac{N_{x}-1}{N_{x}}, & x=y \in(1, \ldots, 2 N) \\ 0, & \text { otherwise }\end{cases}$

where $\lambda_{0}$ is the probability that a person would enter the shadowing area, and mod represents the modulo operation.

To obtain the average PER of each state, we approximate the average received SNR of state $n$ by $\left(\Gamma_{n}+\Gamma_{n+1}\right) / 2$, where $\Gamma_{n}$ corresponds to the SNR at the boundary of zone $n$, provided that there is a sufficient number of partition zones. Given the average received SNR $\gamma_{b}$, the PER that is associated with state $n$, i.e., $\theta_{n}$, is calculated by $\theta_{n}=1-\left(1-\mathrm{BER}_{n}\right)^{L}$, where $\mathrm{BER}_{n}=Q\left(\sqrt{2 \gamma_{b}}\right)$ for antipodal signals [23], and $L$ denotes the packet size. Given the transition probabilities and the average PER that is associated with each state, we obtain a Markov chain that describes the channel variation at the packet level. Note that the above Markov channel model can be formulated as either a discrete or continuous one. Here, we choose to work on a discrete model to facilitate the queuing analysis, as will be presented in Section III. Fig. 2 illustrates how the average received SNR curve is partitioned and the resulting Markov chain.

\section{Queuing Model And Analysis}

To capture the evolution of the joint service/vacation and channel variations under different reservation methods, we develop the analytical model based on the following assumptions and notations.

1) We consider a fixed reservation pattern. A user can reserve time slots using either hard or soft reservation, but not both. The mixed use of both reservations is not considered.

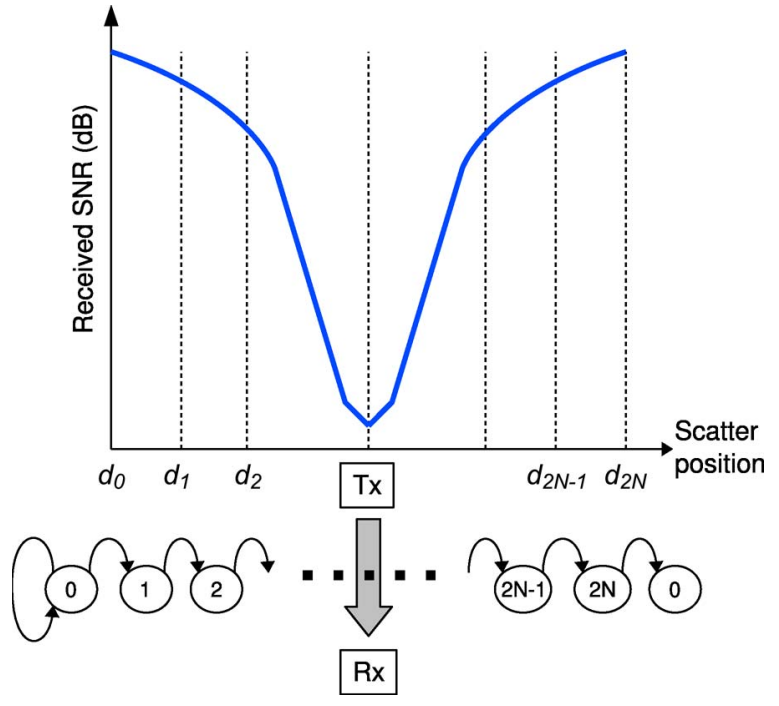

Fig. 2. Partition method for the Markov channel model with a single scatter.

2) The time axis is slotted such that one MAS that is defined in WiMedia MAC is equal to $S$ slots, which serves as the basic time unit of our analysis. For simplicity, at most one packet can be successfully transmitted per slot.

3) An infinite buffer size is assumed. The terms "buffer" and "queue" will be used interchangeably.

4) The tagged user's queue is modeled as a discrete-time queue with vacation. The length of a vacation period $V$, consisting of multiple service periods of other users, follows the discrete-PH-type distribution, which is denoted by $\mathrm{PH}_{d}(\boldsymbol{\eta}, \mathbf{V})$ with order $K$. For simplicity, other periods such as the BP and the PCA period are omitted; however, they can readily be included in the analysis, as explained in Section II-A.

5) One packet may arrive at the beginning of the slot with probability $\alpha$, and one packet may leave at the end of the slot depending on the channel quality, which is represented by the average received SNR $\gamma_{b}$.

6) The UWB channel variation due to shadowing is captured by a discrete-time Markov chain with transition probability matrix $\mathbf{H}=\left[h_{x, y}\right]$ in (3).

7) The average service time is denoted by $1 / \mu$, where $\mu$ is the average service rate.

8) For the soft reservation, whenever the owner finds an empty buffer, it will release the remaining time in the current MAS and resume its transmission in the next reserved MAS. This assumption is justified by the fact that if the buffer becomes empty, it implies that the reserved bandwidth is larger than the actual requirement under the current channel quality and packet arrival rate. Thus, the most efficient use of the bandwidth is achieved by adjusting the amount of the reserved bandwidth (i.e., slots) according to the packet arrival rate and the channel status in real time. The strategy of bandwidth adaptation is out of the scope of this paper. Here, the sender simply releases the reserved slots if an empty buffer is observed and aggregates the packets to transmit in the next reservation. This should serve as the worst-case scenario of using the soft reservation. 
The analysis of the DRP over the UWB shadowing channel is conducted by modeling the joint behaviors of the queue length, the channel state, and the service/vacation period as a discrete-time Markov chain $\chi$ with state space $\{(i,(0, k, x) \cup$ $(s, x))\}$, where $i$ is the number of packets in the queue during vacation (service); in the tuple $(0, k, x), 0$ represents that the tagged user is in the vacation period, $k$ denotes the $\mathrm{PH}$ of the vacation, and $x$ is the channel state; the tuple $(s, x)$ refers to the service state with $s=[1, \ldots, S]$ denoting the slot index. By lexicographically ordering the state space of the Markov chain, the corresponding transition probability matrix $\mathbf{P}$ can be obtained and partitioned as

$$
\mathbf{P}=\left[\begin{array}{ccccccc}
\mathbf{A}_{\mathbf{0}} & \mathbf{C}_{\mathbf{0}} & \mathbf{0} & \mathbf{0} & \mathbf{0} & \ldots & \cdots \\
\mathbf{B}_{\mathbf{0}} & \mathbf{A}_{1} & \mathbf{C}_{1} & \mathbf{0} & \mathbf{0} & \ldots & \ldots \\
\mathbf{0} & \mathbf{B}_{1} & \mathbf{A}_{1} & \mathbf{C}_{1} & \mathbf{0} & \ldots & \ldots \\
\mathbf{0} & \mathbf{0} & \mathbf{B}_{1} & \mathbf{A}_{1} & \mathbf{C}_{1} & \ldots & \ldots \\
\vdots & \vdots & \vdots & \vdots & \vdots & \vdots & \vdots
\end{array}\right]
$$

where each component is a block matrix describing the evolution of the queue length. The derivation of $\mathbf{P}$ is given in Appendix II. It is observed that $\mathbf{P}$ has the exact geometric structure as the quasi-birth and death (QBD) process [24]. In this context, the matrix-geometric solution can readily be used to solve $\mathbf{P}$. To differentiate the underlying reservation method, the Markov chain corresponding to the hard and soft reservations is denoted by $\chi_{h}$ and $\chi_{s}$, respectively. Similarly, we use the notations $\mathbf{P}_{h}$ and $\mathbf{P}_{s}$ to differentiate the transition probability matrices of the hard and soft reservations, respectively.

We note that the above Markov chain model is not limited to the Bernoulli arrival process. For example, a more general Markovian arrival process can be represented by two substochastic matrices and incorporated in embedded Markov chain $\chi$ [18]. In addition, the consideration of one successful transmission per slot implies that the QBD process has onestep memory, as can be observed from (4). Generalization to multiple transmissions per slot simply leads to multistep memories in the QBD process. The model can also be extended to the finite buffer case using the approach in [25].

\section{A. Stationary Probability of $\chi_{h}$}

We resort to the matrix-geometric solution to solve Markov chain $\chi_{h}$. If $\chi_{h}$ is positive recurrent, i.e., the stability condition holds, the matrix-quadratic equation

$$
\mathbf{R}=\mathbf{C}_{0}+\mathbf{R} \mathbf{A}_{1}+\mathbf{R}^{2} \mathbf{B}_{0}
$$

has the minimum nonnegative solution $\mathbf{R}$, with all the eigenvalues within the unit disk. The stationary distribution $\boldsymbol{\pi}=$ $\left[\begin{array}{llll}\boldsymbol{\pi}_{0} & \boldsymbol{\pi}_{1} & \boldsymbol{\pi}_{2} & \ldots\end{array}\right]$ can be expressed as the matrix geometric form

$$
\boldsymbol{\pi}_{k}=\boldsymbol{\pi}_{0} \mathbf{R}^{k}, \quad k \geq 0
$$

where $\pi_{k}$ is the stationary probability of $k$ packets in the queue. From $\boldsymbol{\pi} \mathbf{P}=\boldsymbol{\pi}$, we can obtain a set of linear equations, i.e.,

$$
\boldsymbol{\pi}_{0}\left(\mathbf{A}_{\mathbf{0}}+\mathbf{R B}_{\mathbf{0}}\right)=\boldsymbol{\pi}_{0}
$$

Furthermore, the normalization condition $\boldsymbol{\pi} \mathbf{1}=1$ can be reduced to

$$
\boldsymbol{\pi}_{0}(\mathbf{I}-\mathbf{R})^{-1} \mathbf{1}=1 .
$$

The stationary probability $\boldsymbol{\pi}_{0}$ can be obtained by jointly solving (7) and (8).

\section{B. Stationary Probability of $\chi_{s}$}

In the case of the soft reservation, the number of states for the boundary level is different from that for the nonboundary levels. In this context, the stationary distributions of $\mathbf{P}_{s}$ have the following matrix geometric form [24, p. 24]:

$$
\boldsymbol{\pi}_{k}=\boldsymbol{\pi}_{1} \mathbf{R}^{k-1}, \quad k \geq 2 .
$$

The above equation together with $\boldsymbol{\pi} \mathbf{P}=\boldsymbol{\pi}$ gives a set of linear equations, which is given as

$$
\mathbf{x} B[\mathbf{R}]=\mathbf{x}
$$

where $\mathbf{x}=\left[\begin{array}{ll}\boldsymbol{\pi}_{0} & \boldsymbol{\pi}_{1}\end{array}\right]$, and

$$
B[\mathbf{R}]=\left[\begin{array}{cc}
\mathbf{A}_{\mathbf{0}} & \mathbf{C}_{\mathbf{0}} \\
\mathbf{B}_{\mathbf{0}} & \mathbf{A}_{1}+\mathbf{R B}_{1}
\end{array}\right] .
$$

Since $B[\mathbf{R}]$ is singular, we need other independent equations, which can be obtained from the normalization condition $\boldsymbol{\pi} \mathbf{1}=1$ and reduced to

$$
\boldsymbol{\pi}_{0} \mathbf{1}+\boldsymbol{\pi}_{1}(\mathbf{I}-\mathbf{R})^{-1} \mathbf{1}=1
$$

Then, we can solve (10) and (12) to find $\pi_{0}$ and $\boldsymbol{\pi}_{1}$. Other $\boldsymbol{\pi}_{i}, i \geq 2$ can be obtained from (9).

\section{Performance Metrics}

1) Stability: The stability condition of the vacation queue has been given in [18] for a time-limited polling system. The queuing system that is modeled in this paper is analogous to that in [18], where the system is stable under the following condition.

Proposition 1: The system is stable if

$$
\frac{\alpha(S+\bar{V})}{\mu}<S .
$$

Proof: Define one cycle as the duration between the end of one vacation period and the end of next vacation. Because the user can occupy the channel for at most $S$ slots, regardless of the used reservation method, the average length of one cycle time is $S+\bar{V}$. During one cycle period, the average number of arrivals is $\alpha(S+\bar{V})$. Given the average service time $1 / \mu$, the average time to serve the arrivals during one cycle is $\alpha(S+\bar{V}) / \mu$, which must be less than the maximum service period $S$, for the system to be stable.

2) Mean Service Time: Define the service time of a packet as the duration since a packet is ready to send to the time it is successfully received by the Rx. To simplify the analysis, we assume that the channel quality remains the same during the 
packet transmission period. We will verify this assumption in Section IV.

Suppose that a packet is ready to send at slot $s$ when the channel is in state $x$, for $1 \leq s \leq S$ and $0 \leq x \leq 2 N$, with probability $p_{H}(s, x)$. Because a packet may become the headof-line (HOL) at one of the slots during a MAS with an equal chance, which is independent of the channel state, we have

$$
p_{H}(s, x)=\frac{1}{(2 N+1) S} .
$$

Assuming that the channel remains on same state $x$ during the packet transmission process, the number of time slots that are consumed until a successful transmission follows a geometric distribution with parameter $\theta_{x}$. In other words, a packet may undergo $m$ transmission trials for a successful transmission with probability $\theta_{x}^{m-1}\left(1-\theta_{x}\right)$, and it consumes $m+\lfloor(m-$ 1) $/ S\rfloor \bar{V}$ slots in total. Therefore, we obtain the average service time as

$$
\begin{aligned}
\frac{1}{\mu} & =\sum_{s=1}^{S} \sum_{x=0}^{2 N} p_{H}(s, x) \sum_{m=1}^{\infty} \theta_{x}^{m-1}\left(1-\theta_{x}\right)\left(m+\left\lfloor\frac{m-1}{S}\right\rfloor \bar{V}\right) \\
& =\frac{1}{(2 N+1) S} \sum_{s=1}^{S} \sum_{x=0}^{2 N}\left(\frac{1}{1-\theta_{x}}+\nu\right)
\end{aligned}
$$

where $\nu=\sum_{m=1}^{\infty} \theta_{x}^{m-1}\left(1-\theta_{x}\right)\lfloor(m-1) / S\rfloor \bar{V}$.

3) Average Throughput: The average throughput, which is denoted as $\Omega$, is defined as the average number of successfully transmitted packets per slot. Since packets can only be transmitted during the reservation period, the average throughput is computed as

$$
\Omega=\sum_{i=1}^{\infty} \sum_{x=0}^{2 N} \underbrace{\sum_{s=1}^{S} \pi_{i, s, x}}_{(\star)} c_{x}\left(1-\theta_{x}\right)
$$

where $\pi_{i, s, x}$ represents the joint probability of queue length $i$, channel state $x$, and service epoch $s ; c_{x}$ denotes the maximum number of successful packets per slot given channel state $x$, which is assumed to be one according to our model assumption. In (16), $(\star)$ represents the joint probability that there are $i$ packets in queue during the reservation period with the channel state being $x$, which is given by

$$
(\star)=\boldsymbol{\pi}_{i}\left(\mathbf{q} \otimes \mathbf{1}_{x}\right), \quad \mathbf{q}=[\underbrace{0, \ldots, 0}_{2 N+1} \underbrace{1, \ldots, 1}_{S}]^{T}
$$

where $\mathbf{1}_{x}$ is a column vector of zeros, except for the $x$ th position being one. Using (17), (16) can be written as

$$
\Omega=\sum_{i=1}^{\infty} \boldsymbol{\pi}_{i} \sum_{x=0}^{2 N}\left(\mathbf{q} \otimes \mathbf{1}_{x}\right)\left(1-\theta_{x}\right)=\sum_{i=1}^{\infty} \boldsymbol{\pi}_{i} \mathbf{q} \otimes \hat{\boldsymbol{\Theta}}
$$

where $\hat{\boldsymbol{\Theta}}=\left[1-\theta_{0} 1-\theta_{2} \ldots 1-\theta_{2 N}\right]^{T}$. For the hard reservation, the average throughput is given by

$$
\Omega_{h}=\boldsymbol{\pi}_{0} \mathbf{R}(\mathbf{I}-\mathbf{R})^{-1} \mathbf{q} \otimes \hat{\boldsymbol{\Theta}} .
$$

TABLE I

NUMERICAL PARAMETERS

\begin{tabular}{l|c}
\hline \hline packet size & 1500 bytes \\
\hline slot length $\left(t_{s}\right)$ & $256 \mu \mathrm{s}$ \\
\hline body width $(r)$ & $30 \mathrm{~cm}$ \\
\hline mobility speed $(v)$ & $0.5 \mathrm{~m} / \mathrm{s}$ \\
\hline scatter arrival probability $\left(\lambda_{0}\right)$ & 0.3 \\
\hline scatter-receiver initial distance $(d)$ & $1 \mathrm{~m}$ \\
\hline number of channel states $(N)$ & 21 \\
\hline
\end{tabular}

Similarly, the average throughput of the soft reservation can be given by

$$
\Omega_{s}=\boldsymbol{\pi}_{1}(\mathbf{I}-\mathbf{R})^{-1} \mathbf{q} \otimes \hat{\mathbf{\Theta}} .
$$

4) Mean Waiting Time: For the DRP with the hard reservation, the average queue length at an arbitrary time, which is denoted as $L_{h}$, is derived as

$$
L_{h}=\sum_{k=1}^{\infty} k \boldsymbol{\pi}_{k} \mathbf{1}=\boldsymbol{\pi}_{0} \mathbf{R}(\mathbf{I}-\mathbf{R})^{-2} \mathbf{1} .
$$

Similarly, for the DRP with the soft reservation, the average queue length at an arbitrary time, which is denoted as $L_{s}$, is given by

$$
L_{s}=\boldsymbol{\pi}_{1}(\mathbf{I}-\mathbf{R})^{-2} \mathbf{1} .
$$

Mean waiting time $\bar{W}$ can be obtained by Little's law as

$$
\begin{aligned}
& \bar{W}_{h}=\frac{L_{h}}{\lambda} \\
& \bar{W}_{s}=\frac{L_{s}}{\lambda} .
\end{aligned}
$$

\section{NUMERICAl RESUltS AND Discussions}

This section starts with the simulation setup, including the methodology and the parameters. The analytic and simulation results are compared to validate the mathematical modeling. The interaction between various system parameters and protocol performance will also be discussed.

We first simulate the shadowing UWB channel following the procedures described in Section II-B and obtain the normalized average shadowed power at the Rx end. Table I lists the relevant parameters. A discrete-time Markov chain with 21 states is used to model the channel quality due to the people shadowing process, where the received SNR of each state is approximated by the mean of the SNR of the state boundaries. Based on the obtained packet-level channel model, we simulate the discretetime system described in Section III. The tagged user can access the channel during its reserved MAS of length $S$ slots, whereas the interval between two reservation periods is considered as the vacation time with the length determined by a discrete- $\mathrm{PH}$ distribution. The simulation run time is $5 \times 10^{6}$ time slots. An error-free feedback channel for signaling the acknowledgment is assumed such that the sender can obtain the reception status of the transmitted packet at the end of the time slot.

In the sequel, we demonstrate the analytical results based on the equations presented in Section III. The accuracy of 


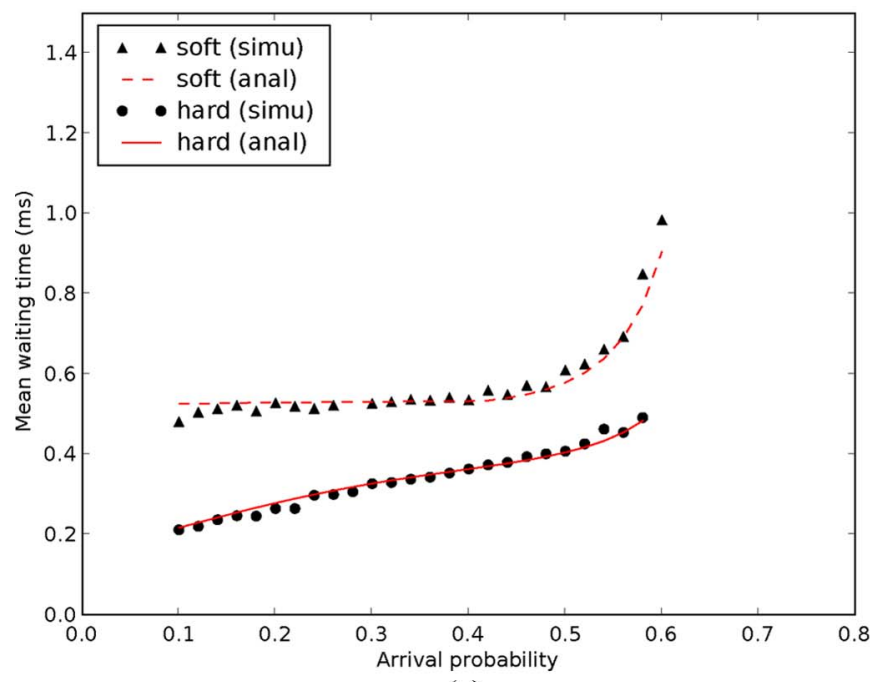

(a)

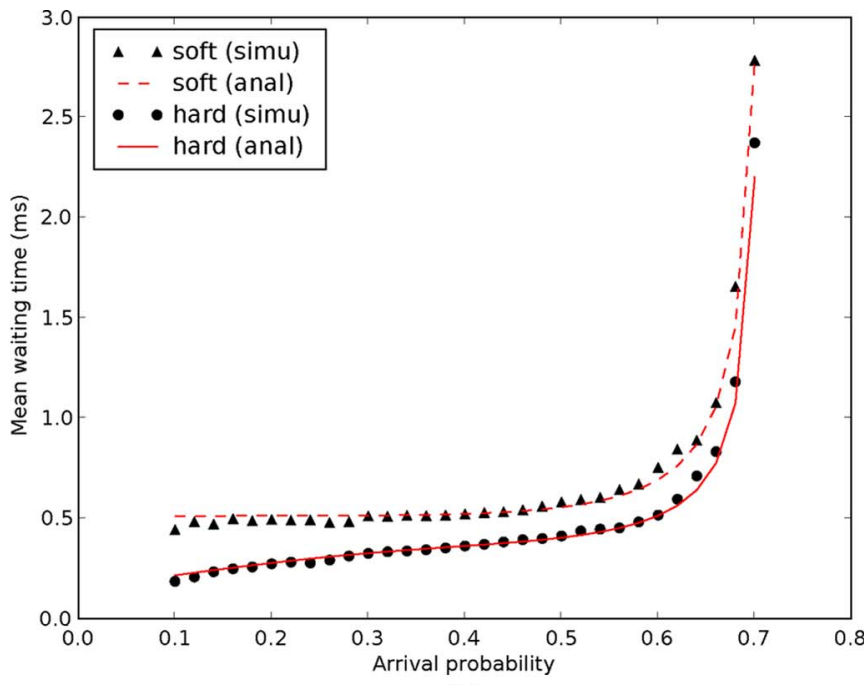

(b)

Fig. 3. Comparison of the mean waiting time of the DRP using hard and soft reservations under a different traffic load and an average received SNR for $S=7$

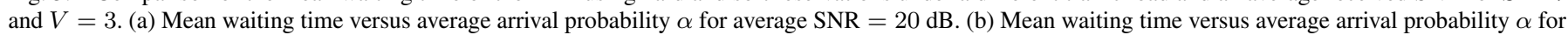
average $\mathrm{SNR}=25 \mathrm{~dB}$.

the analytical model heavily relies on rate matrix $\mathbf{R}$, which is iteratively computed until $\left\|\mathbf{R}^{(k+1)}-\mathbf{R}^{(k)}\right\| \leq 10^{-8}$, where $k$ denotes the iteration index.

\section{A. Mean Waiting Time}

The mean waiting times of the DRP with soft and hard reservations versus the arrival probability under different channel quality, which is represented by the average received SNR without shadowing, are shown in Fig. 3(a) and (b), respectively, where reservation period $S=7$ slots and vacation period $V=$ 3 slots. As seen from the figure, the analytical results match the simulation ones very well. The figure also shows that a higher received SNR reduces the mean waiting time, as expected. Comparing the performance of the DRP with different reservation methods, the soft reservation incurs a higher mean waiting time than that of the hard reservation, and the difference is more significant for a lower received SNR. From the network standpoint, the soft reservation is advantageous for improving the channel utilization by releasing the unused slots to the neighbors. However, this is achieved at the cost of reducing the possible transmission time, during which packets may still arrive but need to wait for the next reservation period. In the hard reservation, these packets may instantly be transmitted within the current reservation period. Hence, there is a tradeoff between the network resource utilization and the QoS of the traffic flow. Although configuring the optimal reservation pattern might not be trivial in a distributed network, a rule of thumb may be to use the soft reservation in a conservative way. A user may release the unused slot if the traffic incoming rate slows down for a period of time, and the number of contending nodes is large. Otherwise, the reserved time slots should be persistently used, even if the buffer is empty. On the other hand, the two reservation methods similarly perform for a high arrival probability region. The reason is obvious since the queue is seldom empty when the packet arrives densely. As a result, slot release can rarely take place, implying that the soft reservation reduces to the hard reservation. Finally, we show the average delay under different reservation patterns by considering two types of reservation patterns. One is resulted from a uniformly spaced reservation pattern, i.e., a constant vacation period that is represented by $V_{1} \sim \mathrm{PH}_{d}\left(\boldsymbol{\eta}_{1}, \mathbf{V}_{1}\right)$, and the other results from a randomly spaced reservation pattern that is driven by a vacation period distribution $V_{2} \sim \mathrm{PH}_{d}\left(\boldsymbol{\eta}_{2}, \mathbf{V}_{2}\right)$. For illustration purposes, we generate the vacation period using two numerical examples, i.e.,

$$
\begin{aligned}
\boldsymbol{\eta}_{1} & =[1,0,0,0] \\
\mathbf{V}_{1} & =\left[\begin{array}{llll}
0 & 1 & 0 & 0 \\
0 & 0 & 1 & 0 \\
0 & 0 & 0 & 1 \\
0 & 0 & 0 & 0
\end{array}\right] \\
\boldsymbol{\eta}_{2} & =\left[\begin{array}{llll}
0.4,0.25,0.2,0.15
\end{array}\right] \\
\mathbf{V}_{2} & =\left[\begin{array}{cccc}
0.2 & 0.3 & 0.25 & 0.25 \\
0 & 0.7 & 0.3 & 0 \\
0 & 0 & 0.5 & 0.3 \\
0 & 0 & 0 & 0
\end{array}\right]
\end{aligned}
$$

and $\bar{V}_{1}=\bar{V}_{2}=4, \operatorname{var}\left[V_{1}\right]=0$, and $\operatorname{var}\left[V_{2}\right]=9.44$. The vacation time with a larger variance implies that the reserved pattern is more random. In Fig. 4, the service period is seven slots long, and the average SNR is equal to $20 \mathrm{~dB}$. For presentation brevity, only analytical results are shown. As seen from Fig. 4(a) for a low-medium packet arrival probability, a larger gap between the two mean waiting time curves of the soft reservation indicates that the soft reservation is more sensitive to the reservation pattern. On the other hand, the mean waiting time of the hard reservation reveals a larger slope, implying that the hard reservation is more sensitive to the traffic intensity. Fig. 4(b) shows that the effect of the variance of the vacation time to both reservation methods tends to be magnified as the traffic load increases. For both reservation methods, a uniformly spaced reservation pattern yields a lower packet delay than that resulted from an unequally spaced reservation pattern. This observation 


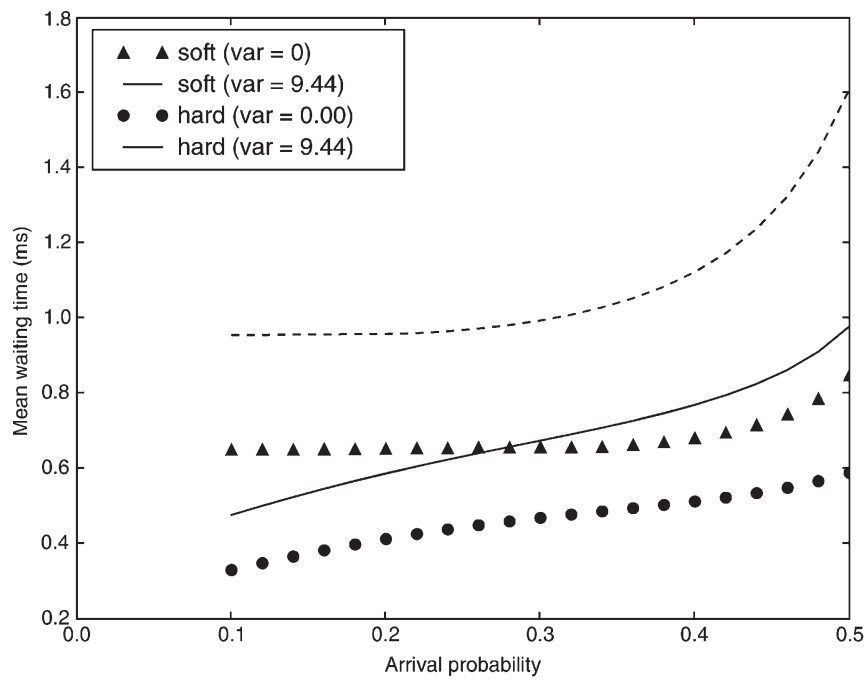

(a)

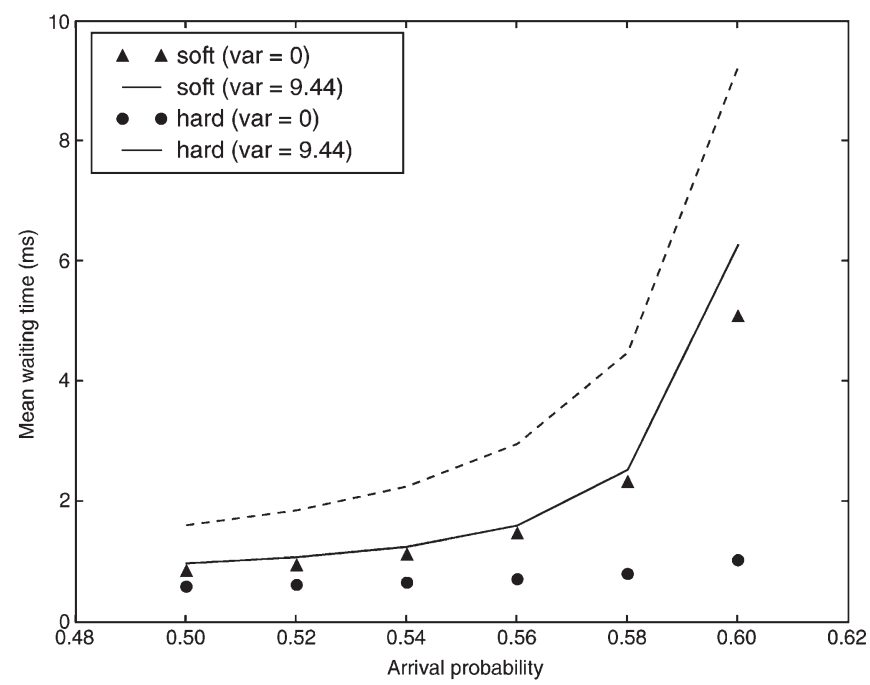

(b)

Fig. 4. Mean waiting time versus arrival probability under different vacation periods $V_{1}(\operatorname{var}=0)$ and $V_{2}(\operatorname{var}=9.44)$ in $(23)$ for $S=7$ and received $\mathrm{SNR}=20 \mathrm{~dB}$. (a) Mean waiting time (in milliseconds) versus average arrival probability $\alpha=0.1-0.5$. (b) Mean waiting time (in milliseconds) versus average arrival probability $\alpha=0.5-0.6$.

basically agrees with previous studies on analyzing the slot allocation pattern in TDMA systems [10], [12], [13]. Although we use numerical examples instead of considering an exact reservation pattern, we elaborate in Appendix I on how the vacation period distribution can be obtained given a specific reservation pattern.

\section{B. Mean Service Time}

The mean service time is the time for a packet to be successfully transmitted through the wireless channel. Intuitively, a higher received SNR provides a lower PER, and thus, a packet can be successfully received with less retransmissions. Table II shows the mean service time (in milliseconds) of the DRP under different channel conditions and vacation distributions. In simulations, we fix traffic intensity $\alpha=0.5$ and service period $S=7$ slots and vary the received SNR and the vacation distributions following (23). Since the two vacation-time distributions
TABLE II

Comparison of the MeAn SERVice Time (In Milliseconds) WiTH DiFFERENT VACATION DISTRIBUTIONS

\begin{tabular}{c|c|c|c}
\hline \hline SNR $(\mathrm{dB})$ & $\begin{array}{c}\text { simulation } \\
\left(V_{1}\right)\end{array}$ & $\begin{array}{c}\text { simulation } \\
\left(V_{2}\right)\end{array}$ & analytical \\
\hline 12 & 0.4539 & 0.4578 & 0.4924 \\
14 & 0.4463 & 0.3833 & 0.3929 \\
16 & 0.3532 & 0.3489 & 0.3511 \\
18 & 0.3080 & 0.3077 & 0.2999 \\
20 & 0.2781 & 0.2794 & 0.2699 \\
22 & 0.2662 & 0.2667 & 0.2611 \\
\hline \hline
\end{tabular}

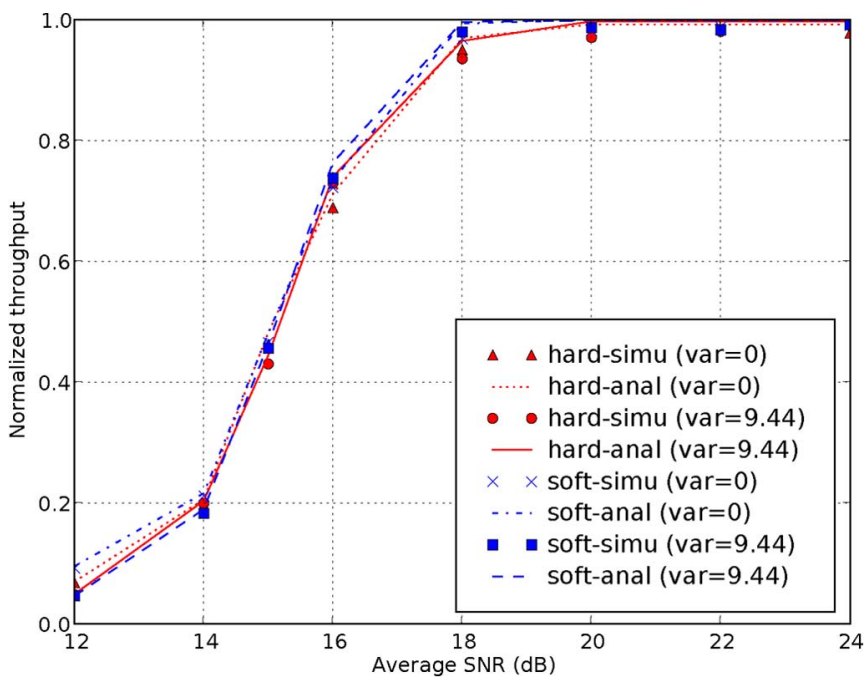

Fig. 5. Normalized throughput of the DRP versus the average SNR with different reservation methods, for $\alpha=0.5$ and $S=7$, and the vacation periods follow (23) with the same mean $\bar{V}=4$ but different variances.

considered in (23) have the same mean, we obtain the same analytical results according to (15). It can be seen that the mean service time is linearly reduced with the increase in the received SNR. In addition, the reservation pattern, whether uniform or interleaved, has nearly a null impact on the mean service time. The agreement between the simulation and analytical results validates the approximated derivation of service time in Section III-C2 based on the assumptions that the channel quality remains the same during the packet transmission period.

\section{Average Throughput}

Here, we present the effects of reservation methods and patterns on average throughput. In Fig. 5, we fix arrival intensity $\alpha=0.5$ and service period $S=7$ slots and consider two vacation distributions following (23), both with the mean vacation period of four slots. In Fig. 5, the horizontal axis represents the average $\mathrm{SNR}$, and the vertical axis represents the average throughput that is normalized by the offered load. Both reservation methods achieve similar throughput and reach their performance limit when the average SNR is sufficiently high. On the other hand, the throughput loss due to shadowing is significant for a low SNR range. In fact, the average throughput without the shadowing effect is close to one, even for received $\mathrm{SNR}=12 \mathrm{~dB}$, which is not shown in the figure for brevity of presentation. To reduce the effect of the shadowing due to moving persons, we may improve the received SNR by employing higher transmission power, lower modulation order, or coding 
rate. Alternatively, in MB-OFDM, where the maximum transmission power is used, time and frequency domain spreading ${ }^{1}$ may be used to increase the SNR of the received data.

\section{CONCLusion}

In this paper, we have proposed an analytical model for the DRP that is defined in WiMedia MAC for a UWB-based WPAN. By embedding the impact of other users in the network to the tagged user via the notion of vacation periods, a discretetime Markovian model for the DRP has been developed. Meanwhile, the dynamics of a UWB channel due to shadowing have also been incorporated in the proposed model that provides a cross-layer performance evaluation of the DRP over UWB networks. We have obtained various important performance metrics in closed-form expressions that allow fast assessment and tuning of protocols. The proposed analytical model has been validated through simulations, and the interactions between several system parameters have been observed from numerical results. It has been shown that the short-term shadowing effect significantly degrades the protocol performance. Comparing the hard and soft reservations, the soft reservation incurs a longer packet delay than that of the hard reservation. As for the effect of the reservation pattern, the soft reservation is more sensitive to the variation in the reservation pattern. Moreover, the traffic intensity has a magnified impact to the nonuniform reservation pattern. Since the shadowing notably degrades the DRP performance, an important future work is to find appropriate adaptive strategies to smoothly support the isochronous traffic.

\section{APPENDIX I \\ Derivation of THE VACATION PERIOd Distribution}

In Section III, the vacation period is represented by a discreet-PH-type distribution $\mathrm{PH}_{d}(\boldsymbol{\eta}, \mathbf{V})$ with order $K$. Based on the derivation in [25], we elaborate how parameters $\boldsymbol{\eta}$ and $\mathbf{V}$ can be obtained using a simple example, where there are five users $\{\mathbf{a}, \mathbf{b}, \mathbf{c}, \mathbf{d}, \mathbf{e}\}$ contending for the channel, and the reservation pattern is given as $\mathbf{a}, \mathbf{b}, \mathbf{c}, \mathbf{a}, \mathbf{e}, \mathbf{c}, \mathbf{d}$. The generalization to an arbitrary number of users and the reservation pattern should be straightforward. We focus on user a. Therefore, the entire reservation pattern can be divided into two subcycles $\phi_{j}, j=1,2$, where $\phi_{1}=\{\mathbf{a}, \mathbf{b}, \mathbf{c}\}$ and $\phi_{2}=\{\mathbf{a}, \mathbf{e}, \mathbf{c}, \mathbf{d}\}$. Let $S_{i}$ denote the length of the reservation period for user $i, i=$ a, ..., e.

We first derive the vacation period length in each subcycle, which is identical to the sum of service periods of other users in the same subcycle. For the hard reservation, the service period is constant with length $S_{i}$ and can be readily characterized by $\mathrm{PH}_{d}\left(\gamma_{i}, \mathbf{F}_{i}\right)$ with order $S_{i}$ given by

$$
\begin{aligned}
\boldsymbol{\gamma}_{i} & =\left[\begin{array}{ll}
1 & \mathbf{0}_{S_{i}-1}
\end{array}\right] \\
\mathbf{F}_{i} & =\left[\begin{array}{cc}
\mathbf{0}_{S_{i}-1} & \mathbf{I}_{S_{i}-1} \\
0 & \mathbf{0}_{S_{i}-1}^{T}
\end{array}\right]
\end{aligned}
$$

\footnotetext{
${ }^{1}$ Time-domain spreading is achieved by transmitting the same information across two consecutive OFDM symbols. Frequency-domain spreading entails transmitting the same information (complex number) on two separate subcarriers within the same OFDM symbol.
}

where $\mathbf{0}_{S_{i}-1}$ is a column vector of all zeros with dimension $S_{i}-1$. For the soft reservation, the service period may be shorter than the reservation period when the buffer becomes empty prior to the expiration of the reservation period. Therefore, the service period is still of the d-PH type, but its derivation is lengthy. See [18, App.] for the details. Let the service period distribution be given and denoted by $\mathrm{PH}_{d}\left(\gamma_{i}, \mathbf{F}_{i}\right)$ for user $i$. Therefore, the vacation period in subcycle $j$ can be represented by $\mathrm{PH}_{d}\left(\boldsymbol{\delta}_{j}, \mathbf{L}_{j}\right)$ with dimension $\sum_{i \in\left\{\phi_{j} \backslash \mathbf{a}\right\}} S_{i}$. In the first subcycle, user a goes on vacation after its service period according to initial probability vector $\boldsymbol{\delta}_{1}$, which is given by $\boldsymbol{\delta}_{1}=\left[\boldsymbol{\gamma}_{\mathbf{b}} \mathbf{0}\right]$, since the service period of user $\mathbf{a}$ is succeeded by that of user $\mathbf{b}$. The channel will be occupied by user $\mathbf{b}$ according to the service period of user $\mathbf{b}$, which is given by $\mathbf{F}_{\mathbf{b}}$. At the end of the service period of user $\mathbf{b}$, user $\mathbf{c}$ will access the channel. Therefore, we have absorption according to $\mathbf{F}_{\mathbf{b}}^{0}$ and beginning of service of user $\mathbf{c}$ according to $\gamma_{\mathbf{c}}$. Then, the channel is occupied by user $\mathbf{c}$ according to $\mathbf{F}_{\mathbf{c}}$. After serving user $\mathbf{c}$, the first subcycle is over according to $\mathbf{F}_{\mathbf{c}}^{0}$. As a result, the vacation period in the first subcycle is completely characterized by

$$
\begin{aligned}
& \mathbf{L}_{1}=\left[\begin{array}{cc}
F_{\mathbf{b}} & \mathbf{F}_{\mathbf{b}}^{0} \gamma_{\mathbf{c}} \\
0 & F_{\mathbf{b}}
\end{array}\right] \\
& \mathbf{L}_{1}^{0}=\left[\begin{array}{c}
\mathbf{0} \\
\mathbf{F}_{\mathbf{c}}^{0}
\end{array}\right] \\
& \boldsymbol{\delta}_{1}=\left[\begin{array}{ll}
\gamma_{\mathbf{b}} & \mathbf{0}
\end{array}\right] .
\end{aligned}
$$

Similarly, the vacation period in the second subcycle $\mathrm{PH}_{d}\left(\boldsymbol{\delta}_{2}, L_{2}\right)$ is given by

$$
\begin{aligned}
\mathbf{L}_{2} & =\left[\begin{array}{ccc}
F_{\mathbf{e}} & \mathbf{F}_{\mathbf{e}}^{0} \gamma_{\mathbf{c}} & 0 \\
0 & F_{\mathbf{c}} & \mathbf{F}_{\mathbf{c}}^{0} \boldsymbol{\gamma}_{\mathbf{d}} \\
0 & 0 & F_{\mathbf{d}}
\end{array}\right] \\
\mathbf{L}_{2}^{0} & =\left[\begin{array}{c}
\mathbf{0} \\
\mathbf{0} \\
\mathbf{F}_{\mathbf{d}}^{0}
\end{array}\right] \\
\boldsymbol{\delta}_{2} & =\left[\begin{array}{lll}
\gamma_{\mathbf{e}} & \mathbf{0} & \mathbf{0}
\end{array}\right] .
\end{aligned}
$$

From the vacation-period distribution in each subcycle, we can construct the vacation-period distribution of the tagged user. It can be seen that the tagged user may take vacation in each subcycle for a certain amount of time then moves to the succeeding subcycle but cannot skip the proceeding subcycle before returning to the current one, and vice versa. The transition between two subcycles can be described by four matrices $\mathbf{U}_{0}, \mathbf{U}_{1}, \mathbf{U}_{2}$, and $\mathbf{U}_{3}$. Matrix $\mathbf{U}_{0}$ specifies the transition during the vacation period in the current subcycle, and $\mathbf{U}_{1}$ determines the end of the current vacation period, which is the next vacation period, and are given by

$$
\begin{aligned}
& \mathbf{U}_{0}=\left[\begin{array}{cc}
\mathbf{L}_{1} & \mathbf{0} \\
\mathbf{0} & \mathbf{L}_{2}
\end{array}\right] \\
& \mathbf{U}_{1}=\left[\begin{array}{cc}
\mathbf{0} & \mathbf{L}_{1}^{0} \boldsymbol{\delta}_{2} \\
\mathbf{L}_{2}^{0} \boldsymbol{\delta}_{1} & 0
\end{array}\right] .
\end{aligned}
$$


The above distribution is interpreted as follows. $\mathbf{U}_{0}(1,1)$ indicates that the tagged user is taking a vacation in the first subcycle according to $\left(\boldsymbol{\delta}_{1}, \mathbf{L}_{1}\right)$. The first subcycle ends according to $\mathbf{L}_{1}^{0}$, and the tagged user enters the second one, where the vacation period is determined by $\left(\boldsymbol{\delta}_{2}, \mathbf{L}_{2}\right)$, as stated by $\mathbf{U}_{1}(1,2)$. Similar arguments lead to positions $\mathbf{U}_{0}(2,2)$ and $\mathbf{U}_{1}(2,1)$. Matrix $\mathbf{U}_{2}$ is used for describing the transitions from the end of the vacation period of the tagged user to the start of the service period, which is given by

$$
\mathbf{U}_{2}=\left[\begin{array}{cc}
\mathbf{0} & \mathbf{L}_{1}^{0} \\
\mathbf{L}_{2}^{0} & \mathbf{0}
\end{array}\right]
$$

Matrix $\mathbf{U}_{3}$ defines the transitions from the service period of the tagged user to the beginning of the vacation period, as given by

$$
\mathbf{U}_{3}=\left[\begin{array}{cc}
\mathbf{0} & \boldsymbol{\delta}_{2} \\
\boldsymbol{\delta}_{1} & \mathbf{0}
\end{array}\right]
$$

Based on the above derivation, we can modify the matrix blocks in (4) as follows.

- Matrix $\mathbf{V}$ is replaced with matrix $\mathbf{U}_{0}$.

- Matrix $\mathbf{V}^{0} \boldsymbol{\eta}$ is replaced with matrix $\mathbf{U}_{1}$.

- Vector $\mathbf{V}^{0}$ is replaced with matrix $\mathbf{U}_{2}$.

- Vector $\boldsymbol{\eta}$ is replaced with matrix $\mathbf{U}_{3}$.

Unavoidably, rate matrix $\mathbf{R}$ becomes larger. Furthermore, the service period duration in the soft reservation depends on the vacation period distribution that involves more computation. The iterative algorithm in [25] with moderate modification can be used to obtain the service period of the tagged user.

\section{APPENDIX II \\ DERIVATION OF MATRIX BLOCKS IN (4)}

In this Appendix, we give the details on how transition probability matrix (4) can be obtained. Appendix II.A deals with the hard reservation, and Appendix II.B considers the soft reservation. Each element in (4) describes the evolution of the queue length, which is driven by the packet arrival probability and the channel status (in terms of the SNR), depending on whether the current slot is occupied by the tagged user (i.e., the service period) or by other users (i.e., the vacation period). We introduce some auxiliary variables: $i$ and $j$ denote the queue lengths for $i, j \geq 0 ; k$ and $l$ represent the vacation PHs for $k, l \in(1, \ldots, K) ; x$ and $y$ represent the channel states for $x, y \in(0, \ldots, 2 N)$; and $s \in(1, \ldots, S)$ denotes the slot index. Meanwhile, the following matrix shall be used frequently:

$$
\begin{aligned}
\boldsymbol{\Theta} & =\operatorname{diag}\left(\theta_{0}, \theta_{2}, \ldots, \theta_{2 N}\right) \\
\boldsymbol{\Theta}^{\prime} & =\mathbf{I}_{2 N+1}-\boldsymbol{\Theta}
\end{aligned}
$$

and $\mathbf{I}_{2 N+1}$ is an identity matrix of dimension $2 N+1$.

\section{A. Markov Chain for the DRP With the Hard Reservation}

For the DRP with the hard reservation, the tagged user persistently occupies the service period, regardless of the buffer status. Each block in $\mathbf{P}_{h}$ is a square matrix of order $(2 N+$ $1)(K+S) \times(2 N+1)(K+S)$, as detailed in the following.
1) $\mathbf{A}_{0}$ : The queue length remains zero in the following situations.

a) During the vacation period, the system state switches from $\{0,(0, k, x)\}$ to $\{0,(0, l, y)\}$ with probability $(1-$ $\alpha) v_{k, l} h_{x, y}$. The transition probability of dimension $(2 N+1) K \times(2 N+1) K$ is given as

$$
\mathbf{A}_{\mathbf{0}}^{\mathbf{3}}=(1-\alpha) \mathbf{V} \otimes \mathbf{H}
$$

where $\otimes$ denotes the Kronecker product.

b) The transition from the vacation period to the service period, i.e., from state $\{0,(0, k, x)\}$ to $\{0,(s, y)\}$, occurs with probability $(1-\alpha) v_{k}^{0} h_{x, y}$ for $s=1$; otherwise, it is zero. The transition probability matrix of dimension $(2 N+1) K \times(2 N+1)$ for the former case can be represented as

$$
\mathbf{A}_{\mathbf{0}}^{\mathbf{2}}=(1-\alpha) \mathbf{V}^{\mathbf{0}} \otimes \mathbf{H} .
$$

c) The transition during the service period only occurs from state $\{0,(s, x)\}$ to $\{0,(s+1, y)\}$ for $0 \leq s \leq S-1$ with probability $(1-\alpha) h_{x, y}+\alpha\left(1-\theta_{x}\right) h_{x, y}$, which can be written in the matrix form of dimension $(2 N+1) \times$ $(2 N+1)$ as

$$
\mathbf{A}_{\mathbf{0}}^{\mathbf{0}}=(1-\alpha) \mathbf{H}+\alpha \mathbf{\Theta}^{\prime} \mathbf{H} .
$$

d) At the end of the service period, system state $\{0,(S, x)\}$ may transit to $\{0,(0, k, y)\}$ with probability $(1-$ $\alpha) \eta_{k} h_{x, y}+\alpha\left(1-\theta_{x}\right) \eta_{k} h_{x, y}$. Thus, we have the block matrix of dimension $(2 N+1) \times(2 N+1) K$, i.e.,

$$
\mathbf{A}_{\mathbf{0}}^{\mathbf{1}}=(1-\alpha) \boldsymbol{\eta} \otimes \mathbf{H}+\alpha \boldsymbol{\eta} \otimes \boldsymbol{\Theta}^{\prime} \mathbf{H} .
$$

The resultant transition probability matrix that describes the queue remaining zero in two consecutive slots has the following structure:

$$
\mathbf{A}_{0}=\left[\begin{array}{cccccc}
\mathbf{A}_{\mathbf{0}}^{\mathbf{3}} & \mathbf{A}_{\mathbf{0}}^{2} & \mathbf{0} & \ldots & \ldots & \cdots \\
\mathbf{0} & \mathbf{0} & \mathbf{A}_{\mathbf{0}}^{\mathbf{0}} & \mathbf{0} & \ldots & \cdots \\
\vdots & \vdots & \ddots & \mathbf{A}_{1}^{0} & \ldots & \ldots \\
\vdots & \vdots & \vdots & \ddots & \ddots & \ldots \\
\vdots & \vdots & \vdots & \vdots & \ddots & \mathbf{A}_{\mathbf{0}}^{0} \\
\mathbf{A}_{\mathbf{0}}^{1} & \cdots & \cdots & \cdots & \cdots & \mathbf{0}
\end{array}\right]
$$

Using similar argument, we can obtain $\mathbf{A}_{\mathbf{1}}$ with the same structure as $\mathbf{A}_{\mathbf{0}}$, but with different components, as follows:

$$
\begin{aligned}
& \mathbf{A}_{\mathbf{1}}^{\mathbf{3}}=(1-\alpha) \mathbf{V} \otimes \mathbf{H}, \mathbf{A}_{\mathbf{1}}^{\mathbf{2}}=(1-\alpha) \mathbf{V}^{0} \otimes \mathbf{H} \\
& \mathbf{A}_{\mathbf{1}}^{\mathbf{1}}=(1-\alpha) \boldsymbol{\eta} \otimes \mathbf{\Theta} \mathbf{H}+\alpha \boldsymbol{\eta} \otimes \boldsymbol{\Theta}^{\prime} \mathbf{H} \\
& \mathbf{A}_{\mathbf{1}}^{\mathbf{0}}=(1-\alpha) \mathbf{\Theta} \mathbf{H}+\alpha \boldsymbol{\Theta}^{\prime} \mathbf{H} .
\end{aligned}
$$

In addition, $\mathbf{C}_{\mathbf{0}}$ is given by

$$
\begin{aligned}
& \mathbf{C}_{\mathbf{0}}^{\mathbf{3}}=\alpha \mathbf{V} \otimes \mathbf{H} \\
& \mathbf{C}_{\mathbf{0}}^{\mathbf{2}}=\alpha \mathbf{V}^{\mathbf{0}} \otimes \mathbf{H} \\
& \mathbf{C}_{0}^{1}=\alpha \boldsymbol{\eta} \otimes \boldsymbol{\Theta H} \\
& \mathbf{C}_{\mathbf{0}}^{\mathbf{0}}=\alpha \boldsymbol{\Theta} \mathbf{H} .
\end{aligned}
$$


Transition probability matrix $\mathbf{B}_{\mathbf{1}}$, corresponding to the queue length decreasing by one, has the following structure:

$$
\left[\begin{array}{cccccc}
\mathbf{0} & \mathbf{0} & \mathbf{0} & \cdots & \cdots & \cdots \\
\mathbf{0} & \mathbf{0} & \mathbf{B}_{\mathbf{1}}^{\mathbf{0}} & \mathbf{0} & \cdots & \cdots \\
\vdots & \vdots & \ddots & \mathbf{0} & \ldots & \cdots \\
\vdots & \vdots & \vdots & \ddots & \ddots & \cdots \\
\vdots & \vdots & \vdots & \vdots & \ddots & \mathbf{B}_{1}^{0} \\
\mathbf{B}_{1}^{1} & \cdots & \cdots & \cdots & \cdots & \mathbf{0}
\end{array}\right]
$$

with the following components:

$$
\begin{aligned}
& \mathbf{B}_{\mathbf{1}}^{\mathbf{0}}=(1-\alpha) \boldsymbol{\Theta}^{\prime} \mathbf{H} \\
& \mathbf{B}_{\mathbf{1}}^{\mathbf{1}}=(1-\alpha) \boldsymbol{\eta} \otimes \boldsymbol{\Theta}^{\prime} \mathbf{H} .
\end{aligned}
$$

Notice that $\mathbf{B}_{\mathbf{0}}$ is identical to $\mathbf{B}_{\mathbf{1}}$ in the hard reservation case.

\section{B. Markov Chain for the DRP With the Soft Reservation}

The main difference of the soft reservation from the hard reservation is that whenever the tagged user's buffer becomes empty, the unused time slots will be used by other users following the PCA access rule. In other words, the tagged user enters the vacation period if the service period ends or if the queue becomes empty, whichever happens first. Consequently, the system behavior of the soft reservation differs from that of the hard reservation when it involves the queue length being zero, namely, $\mathbf{A}_{\mathbf{0}}, \mathbf{B}_{\mathbf{0}}$, and $\mathbf{C}_{\mathbf{0}}$ in $\mathbf{P}_{\mathbf{s}}$.

1) $\mathbf{A}_{\mathbf{0}}$ : The system remains on state 0 , i.e., the queue length is equal to zero, only when the server is on vacation, and there is no packet arrival. The corresponding transition matrix can be derived as $(1-\alpha)(\mathbf{V} \otimes \mathbf{H})$ before the vacation $\mathrm{PH}$ reaches the absorbing state. For the case that the system enters to the absorbing state, the vacation $\mathrm{PH}$ will be renewed since the queue is still empty, which forces the system to proceed with another vacation time. This will happen with the probability determined by $\mathbf{V}^{\mathbf{0}}$, whereas the new vacation $\mathrm{PH}$ is determined by $\boldsymbol{\eta}$. Overall, block matrix $\mathbf{A}_{\mathbf{0}}$ with dimension $(2 N+1) K \times$ $(2 N+1) K$ is given as

$$
\mathbf{A}_{\mathbf{0}}=(1-\alpha)\left(\mathbf{V} \otimes \mathbf{H}+\mathbf{V}^{\mathbf{0}} \boldsymbol{\eta} \otimes \mathbf{H}\right) .
$$

2) $\mathbf{C}_{\mathbf{0}}$ : The queue length may increase from zero to one during the vacation period with corresponding transition matrix $\alpha \mathbf{V} \otimes \mathbf{H}$. It may also occur when a packet arrives at the end of the vacation period with transition matrix $\alpha \mathbf{1}_{1}^{T} \otimes \mathbf{V}^{\mathbf{0}} \otimes \mathbf{H}$ with dimension $(2 N+1) K \times(2 N+1) S$, where $\mathbf{1}_{1}$ is the column vector, which has 1 in the first position and 0 elsewhere, and $T$ denotes the matrix transpose. As a result, block matrix $\mathbf{C}_{\mathbf{0}}$ can be given by

$$
\mathbf{C}_{\mathbf{0}}=\left[\alpha \mathbf{V} \otimes \mathbf{H} \quad \alpha \mathbf{1}_{1}^{T} \otimes \mathbf{V}^{\mathbf{0}} \otimes \mathbf{H}\right]
$$

with dimension $(2 N+1) S \times(2 N+1)(K+S)$.

3) $\mathbf{B}_{\mathbf{0}}$ : During the vacation period, the probability that the queue length changes from one to zero is zero, and it is $(1-\alpha)\left(1-\theta_{x}\right) \eta_{k} h_{x, y}$ when the system state switches from $(1,(s, x))$ to $(0,(0, k, y))$. Thus, we have block matrix $\mathbf{B}_{0}$ of dimension $(2 N+1)(K+S) \times(2 N+1) K$ as follows:

$$
\mathbf{B}_{\mathbf{0}}=\left[\begin{array}{c}
\mathbf{0} \\
\mathbf{1} \otimes(1-\alpha) \boldsymbol{\eta} \otimes \boldsymbol{\Theta}^{\prime} \mathbf{H}
\end{array}\right]
$$

\section{ACKNOWLEDGMENT}

The authors would like to thank Dr. L. B. Le for his valuable suggestions on attaining the numerical results for the QBD process.

\section{REFERENCES}

[1] M. Z. Win and R. Scholtz, "Ultra-wide bandwidth time-hopping spreadspectrum impulse radio for wireless multiple-access communications," IEEE Trans. Commun., vol. 48, no. 4, pp. 679-689, Apr. 2000.

[2] W. Zhuang, X. Shen, and Q. Bi, "Ultra-wideband wireless communications," Wirel. Commun. Mob. Comput., vol. 3, no. 6, pp. 663-685, Sep. 2003.

[3] C. Duan, G. Pekhteryev, J. Fang, Y. Nakache, J. Zhang, K. Tajima, Y. Nishioka, and H. Hirai, "Transmitting multiple HD video streams over UWB links," in Proc. IEEE CCNC, Las Vegas, NV, Jan. 2006, pp. 691-695.

[4] X. Shen, W. Zhuang, H. Jiang, and J. Cai, "Medium access control in ultra-wideband wireless networks," IEEE Trans. Veh. Technol., vol. 54, no. 5, pp. 1663-1677, Sep. 2005.

[5] L. X. Cai, L. Cai, X. Shen, and J. W. Mark, "Optimizing distributed MAC protocol for multi-hop ultra-wideband wireless networks," in Proc. IEEE INFOCOM, Phoenix, AZ, Apr. 2008, pp. 1912-1920.

[6] High Rate Ultra Wideband PHY and MAC Standard, ECMA International Std. ECMA-368, Dec. 2005. [Online]. Available: http://www. ecma-international.org/publications/standards/Ecma-368.htm

[7] D. Chen, D. Gu, and J. Zbang, "Supporting real-time traffic with QoS in IEEE 802.11e based home networks," in Proc. IEEE CCNC, Las Vegas, NV, Jan. 2004, pp. 205-209.

[8] K.-H. Liu, X. Ling, X. Shen, and J. Mark, "Performance analysis of prioritized MAC in UWB WPAN with bursty multimedia traffic," IEEE Trans. Veh. Technol., vol. 57, no. 4, pp. 2462-2473, Jul. 2008.

[9] D. Wong, F. Chin, M. Shajan, and Y. Chew, "Performance analysis of saturated throughput of PCA in the presence of hard DRPs in WiMedia MAC," in Proc. IEEE WCNC, Hong Kong, Mar. 2007, pp. 423-429.

[10] I. Rubin and Z. Zhang, "Message delay and queue-size analysis for circuit-switched TDMA systems," IEEE Trans. Commun., vol. 39, no. 6, pp. 905-914, Jun. 1991.

[11] M. F. Neuts, J. Guo, M. Zukerman, and H. L. Vu, "The waiting time distribution for a TDMA model with a finite buffer," in Proc. IEEE INFOCOM, San Francisco, CA, Mar. 2003, pp. 177-185.

[12] M. K. Khan and H. Peyravi, "Delay and jitter analysis of generalized demand-assignment multiple access (DAMA) protocols with general traffic," in Proc. 38th Annu. HICSS, Big Island, HI, Jan. 2005, p. 304a.

[13] H. Wu, Y. Xia, and Q. Zhang, "Delay analysis of DRP in MBOA UWB MAC," in Proc. IEEE ICC, Istanbul, Turkey, Jun. 2006, pp. 229-233.

[14] M. Zorzi, A. Chockalingam, and R. Rao, "Throughput analysis of TCP on channels with memory," IEEE J. Sel. Areas Commun., vol. 18, no. 7 , pp. 1289-1300, Jul. 2000 .

[15] H. Rutagemwa and X. Shen, "Modeling and analysis of WAP performance over wireless links," IEEE Trans. Mobile Comput., vol. 2, no. 3, pp. 221232, Jul.-Sep. 2003.

[16] L. Le, E. Hossain, and A. Alfa, "Queuing analysis for radio link level scheduling in a multi-rate TDMA wireless network," in Proc. IEEE Globecom, Dallas, TX, Nov./Dec. 2004, pp. 4061-4065.

[17] H. Shen, L. Cai, and X. Shen, "Performance analysis of TFRC over wireless link with truncated link-level ARQ," IEEE Trans. Wireless Commun., vol. 5, no. 6, pp. 1479-1487, Jun. 2006.

[18] A. S. Alfa, "A discrete MAP/PH/1 queue with vacations and exhaustive time-limited service," Oper. Res. Lett., vol. 18, no. 1, pp. 31-40, Aug. 1995

[19] IEEE 802.15.SG.3a, Channel Modeling Sub-Committee Report Final, IEEE Std. 802.15-02/490rl-SG3a, Feb. 2005.

[20] R. Zhang and L. Cai, "A Markov model for ultra-wideband indoor channel with shadowing," Mobile Networks and Applications (MONET): Special 
Issues on Mobility of Systems, Users, Data, and Computing, vol. 12, no. 5-6, pp. 438-449, Dec. 2007.

[21] A. F. Molisch, Time Variance for UWB Wireless Channels, IEEE Std. P802.15-02/461-SG3a and IEEE Std. 802.15-02/462-SG3a.

[22] S. V. Schell, Analysis of Time Variance of a UWB Propagation Channel, IEEE Std. P802.15-02/452-SG3a and IEEE Std. 802.15-02/453-SG3a.

[23] W.-C. Liu and L.-C. Wang, "BER analysis in a generalized UWB frequency selective fading channel with random arriving clusters and rays," in Proc. IEEE ICC, Glasgow, U.K., Jun. 2007, pp. 4281-4286.

[24] M. F. Neuts, Matrix-Geometric Solutions in Stochastic Models-An Algorithmic Approach. Mineola, NY: Dover, 1994

[25] I. Frigui and A. S. Alfa, "Analysis of a discrete time table polling system with MAP input and time-limited service discipline," Telecommun. Syst., vol. 12, no. 1, pp. 51-77, Aug. 1999.

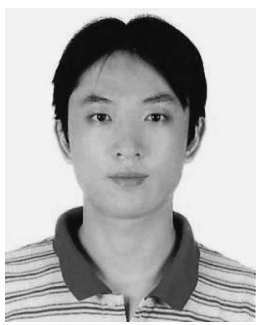

Kuang-Hao Liu (M'08) received the B.S. degree in applied mathematics from National Chiao Tung University, Hsinchu, Taiwan, in 1998 and the M.S. degree in electrical engineering from National Chun Hsing University, Taichung, Taiwan, in 2000.

From 2000 to 2002, he was a Software Engineer with Siemens Telecommunications System Ltd., Taiwan. Since August 2008, he has been with the Department of Electrical Engineering, National Cheng Kung University, Tainan, Taiwan, as an assistant professor. His research interests include UWB communications in personal area networks, resource-allocation problems in wireless networks, and performance analysis for communication protocols.

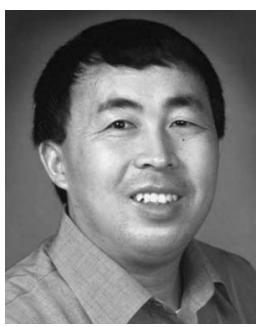

Xuemin (Sherman) Shen (M'97-SM'02) received the B.Sc. degree from Dalian Maritime University, Dalian, China, in 1982 and the M.Sc. and Ph.D. degrees from Rutgers University, Camden, NJ, in 1987 and 1990, respectively, all in electrical engineering.

$\mathrm{He}$ is currently with the Department of Electrical and Computer Engineering, University of Waterloo, Waterloo, ON, Canada, where he is a Professor and the Associate Chair for Graduate Studies. He has coauthored three books and published more than 300 papers and book chapters in wireless communications and networks, control, and filtering. His research focuses on mobility and resource management in interconnected wireless/wired networks, UWB wireless communications systems, wireless security, and ad hoc and sensor networks.

Dr. Shen serves as the Technical Program Chair for many conferences, including the IEEE 2007 Global Telecommunications Conference. He also serves as the Editor/Associate Editor/Guest Editor for the IEEE TRANSACTIONS ON WiRELESS COMMUNICATIONS, the IEEE TRANSACTIONS ON VEHICULAR TECHNOLOGy, Computer Networks, ACM/Wireless Networks, IEEE JOURNAL on Selected AREAS in COMmunications, IEEE Wireless Communications, IEEE Communications Magazine, etc. He was the recipient of the 2002 and 2004 Outstanding Performance Award from the University of Waterloo for outstanding contribution in teaching, scholarship, and service and the 2003 Premier's Research Excellence Award from the Province of Ontario for demonstrated excellence of scientific and academic contributions. He is a Registered Professional Engineer in the Province of Ontario.

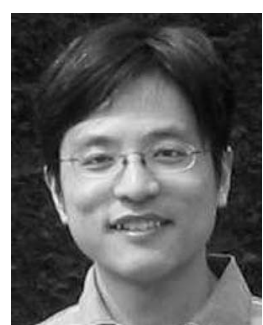

Ruonan Zhang received the B.S. and M.S. degrees in electrical engineering from Xi' an Jiaotong University, Xi' an, China, in 2000 and 2003, respectively. $\mathrm{He}$ is currently working toward the Ph.D. degree with the Department of Electrical and Computer Engineering, University of Victoria, Victoria, BC, Canada.

From 2003 to 2006, he was with Motorola Inc. and, later, with Freescale Semiconductor Inc., Tianjin, China, working on IC architecture and application design. His current research interests include cross-layer design and optimization for wireless networks and WPANs.

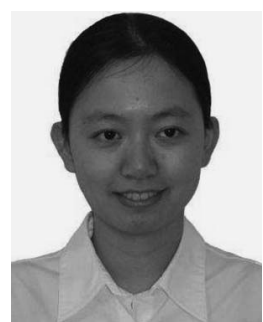

Lin Cai (S'00-M'06) received the M.A.Sc. and $\mathrm{Ph} . \mathrm{D}$. degrees in electrical and computer engineering from the University of Waterloo, Waterloo, ON, Canada, in 2002 and 2005, respectively.

Since July 2005, she has been an Assistant Professor with the Department of Electrical and Computer Engineering, University of Victoria, Victoria, BC, Canada. Her research interests span several areas in wireless communications and networking, with a focus on network protocol and architecture design supporting emerging multimedia traffic over wireless, mobile, ad hoc, and sensor networks.

Dr. Cai serves as an Associate Editor for the EURASIP Journal on Wireless Communications and Networking and the International Journal of Sensor Networks since 2006 and the IEEE TRANSACTIONS ON VEHICULAR TECHNOLOGY since 2007. She was the recipient of the Outstanding Achievement in Graduate Studies Award. 